\title{
Cross-layer Navigation Convolutional Neural Network for Fine-grained Visual Classification
}

\author{
Chenyu Guo ${ }^{1}$, Jiyang Xie ${ }^{1}$, Kongming Liang ${ }^{1}$, Xian Sun ${ }^{2}$, Zhanyu Ma ${ }^{1,3}$ \\ ${ }^{1}$ Pattern Recognition and Intelligent System Lab., Beijing University of Posts and Telecommunications, Beijing, China \\ ${ }^{2}$ Aerospace Information Research Institute, Chinese Academy of Sciences, Beijing, China \\ ${ }^{3}$ Beijing Academy of Artificial Intelligence, Beijing, China
}

\begin{abstract}
Fine-grained visual classification (FGVC) aims to classify sub-classes of objects in the same super-class (e.g., species of birds, models of cars). For the FGVC tasks, the essential solution is to find discriminative subtle information of the target from local regions. Traditional FGVC models preferred to use the refined features, i.e., high-level semantic information for recognition and rarely use low-level information. However, it turns out that low-level information which contains rich detail information also has effect on improving performance. Therefore, in this paper, we propose cross-layer navigation convolutional neural network for feature fusion. First, the feature maps extracted by the backbone network are fed into a convolutional long short-term memory model sequentially from high-level to low-level to perform feature aggregation. Then, attention mechanisms are used after feature fusion to extract spatial and channel information while linking the high-level semantic information and the low-level texture features, which can better locate the discriminative regions for the FGVC. In the experiments, three commonly used FGVC datasets, including CUB-200-2011, Stanford-Cars, and FGVC-Aircraft datasets, are used for evaluation and we demonstrate the superiority of the proposed method by comparing it with other referred FGVC methods to show that this method achieves superior results. https://github.com/PRIS-CV/CN-CNN.git
\end{abstract}

\section{KEYWORDS}

Computer vision, fine-grained visual classification, cross-layer navigation, convolutional long short-term memory, attention mechanism

\section{ACM Reference Format:}

Chenyu Guo ${ }^{1}$, Jiyang $\mathrm{Xie}^{1}$, Kongming Liang ${ }^{1}, \mathrm{Xian}_{\mathrm{Sun}}{ }^{2}$, Zhanyu Ma ${ }^{1,3}$. 2021. Cross-layer Navigation Convolutional Neural Network for Fine-grained Visual Classification. In ACM Multimedia Asia (MMAsia '21), December 13, 2021, Gold Coast, Australia. ACM, New York, NY, USA, 5 pages. https: //doi.org/10.1145/3469877.3490579

\section{INTRODUCTION}

Fine-grained visual classification (FGVC) works to distinguish subclasses of a common visual super-class (e.g., species of birds, models of cars, etc.). The difference of this task from the general image

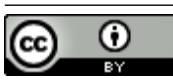

This work is licensed under a Creative Commons Attribution International 4.0 License.

MMAsia '21, December 1-3, 2021, Gold Coast, Australia

(c) 2021 Copyright held by the owner/author(s).

ACM ISBN 978-1-4503-8607-4/21/12.

https://doi.org/10.1145/3469877.3490579 classification lies in the finer granularity of the classes to which the image belongs. Since different sub-classes differ from each other only in subtle ways, the FGVC tasks face two challenges, i.e., large intra-class variations and small inter-class difference. Hence, the commonly accepted solution is to mine discriminative information as much as possible from local regions for the FGVC tasks.

In this case, in the early work, researchers introduced strong supervisions to mine more discriminative information which requires the manual annotation information of the images, for example, object bounding boxes and part annotations $[1,12,30]$. Such methods first locate distinct regions based on additional annotation information, and then extract features from each of them. However, the fine-grained manual annotations of images are expensive and these methods are rarely used in practice. Subsequently, weakly supervision methods which we used in this paper locate discriminative regions with only class labels.

One of the representative class of methods used localizationclassification subnetworks $[3,8,20,28]$ since every part of the object is important for learning discriminative information in the FGVC. They mainly locate discriminative regions through learning part detectors [32], attention mechanisms [4, 7, 9, 24, 31, 33], or filters/activations $[13,28]$. Among them, the attention mechanism is widely used and the effect is very good. Many models like $[5,15,22]$ attempt to incorporate attention mechanisms to improve the performance of CNNs in FGVC tasks. For example, the spatial transformation Network (STN) [15] proposed a learnable module that can be used to locate the most relevant regions in an image to the spatial domain, thus ignoring information from other weakly relevant less important regions. In addition, one way to learn discriminative regions is to integrate multi-level features and guide the features mutually by different levels [7].

Although the above study reports excellent results, the low-level information is sort of overlooked. As the depth of the network increases, the network pays more attention to global high-level abstract semantic information and some low-level detailed information is inescapably lost. In addition, spatio-temporal correlation between layers can refine the process of learning discriminative regions better. Thus, we introduce the ConvLSTM module which can effectively enhance this kind of correlation. In this paper, we propose cross-layer navigation convolutional neural network $(\mathrm{CN}-$ $\mathrm{CNN}$ ), which aims to strengthen the connection between high-level (i.e., semantic information) and low-level (i.e., detailed information) and leverage them for better recognition. First, the feature maps extracted by the backbone network are fed into a convolutional long short-term memory (ConvLSTM) model sequentially from high-level to low-level to perform feature aggregation. Attention mechanisms are used after the feature fusion to extract spatial and 


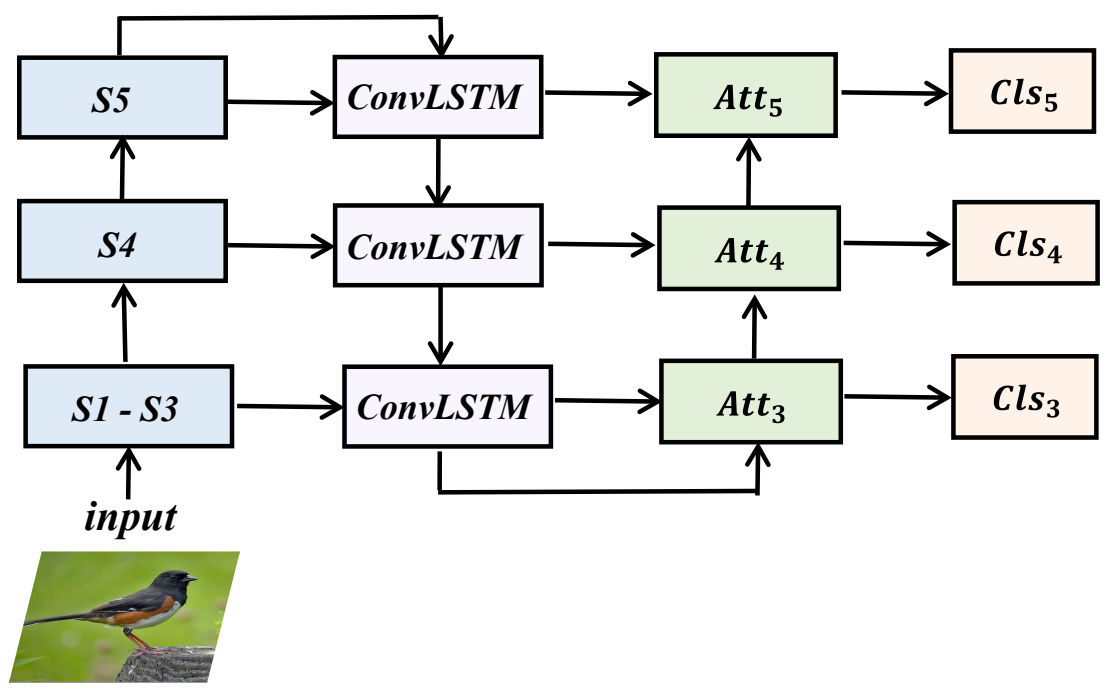

Figure 1: Overall structure of the proposed CN-CNN. Two-pathway Hierarchy are introduced for cross-layer feature navigation in the FGVC. "S1"-"S5" mean stages in the backbone. "Att $i$ " and "Cls ${ }_{i}$ " indicate the attention mechanisms in the LH pathway and the classifiers.
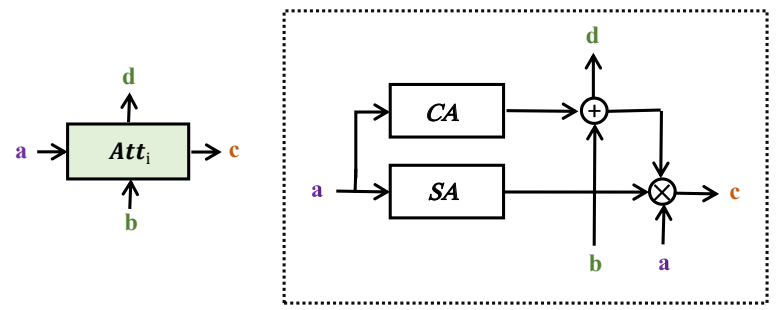

Figure 2: Structure of the attention mechanisms.

channel information while linking high-level semantic information and low-level texture features, which can better locate the discriminative regions for the FGVC.

The major contributions of this work are summarized as follow:

- We propose the $\mathrm{CN}-\mathrm{CNN}$, which aims to strengthen the connection between high-level (i.e., semantic information) and low-level (i.e., detailed information) and leverage them for better recognition by ConvLSTM and attention mechanisms.

- Experimental results on three common FGVC datasets, including CUB-200-2011 [25], Stanford-Cars [16], and FGVCAircraft [21] datasets show that our proposed method can effectively improve the accuracy in the FGVC.

\section{NETWORK STRUCTURE}

In this section, we introduce the network architecture of proposed method in detail. The overall structure of is shown in Figure 1.

\subsection{Two-pathway Hierarchy for Cross-layer Navigation}

In this section, we use ResNet50 as the backbone for the feature extractor. The feature maps obtained by the third, fourth, and fifth stages are used as the input of the cross-layer navigation, noted as S3, S4, and S5, respectively. Two pathways are proposed, including high-low (HL) pathway, which navigates lower-level features by higher-level ones, and low-high (LH) pathway, which refines higherlevel features with lower-level ones.

From high-level to low-level: The feature maps of different stages are sequentially inputted into ConvLSTM for the guidance from high-level to low-level. The features are aggregated to supervise the underlying texture features using the higher-level semantic features.

From low-level to high-level: The low-level feature maps then navigate the high-level ones by generating attention maps for them. In the right attention module, there also exists a pathway from low-level to high-level, which is used to deliver the information from the lower layers to the higher ones and link the channels better.

By this two pathway hierarchy structure, the network can well fuse the semantic feature information of the higher-level with the detail information of the lower-level, simultaneously.

Classifier: Finally, the feature maps $M_{i}^{\prime}$ learned from the two pathway are then fed into three independent classifiers for the FGVC. Each classifier contains a global average pooling (GAP) and two FC layers. The feature maps $M_{i}^{\prime}$ obtained by the LH pathway are then fed into the three layers to obtain predictions. And the final classification results are obtained by averaging predictions.

$$
M_{i}^{\prime}=M_{i} \cdot(S A \oplus C A)
$$


Table 1: Comparison with state-of-the-art methods on three FGVC datasets. The best results are highlighted in bold.

\begin{tabular}{ccccc}
\hline \hline \multirow{2}{*}{ Method } & \multirow{2}{*}{ Base } & \multicolumn{3}{c}{ Datasets } \\
\cline { 3 - 5 } & & CUB-200-2011 & Stanford Cars & FGVC-Aircraft \\
\hline FT VGGNet (CVPR 18) [26] & VGG19 & 77.8 & 84.9 & 84.8 \\
FT ResNet (CVPR 18) [26] & ResNet50 & 84.1 & 91.7 & 88.5 \\
B-CNN (ICCV 15) [18] & VGG16 & 84.1 & 91.3 & 84.1 \\
MA-CNN (ICCV 17) [33] & VGG19 & 86.5 & 92.8 & 89.9 \\
NTS (ECCV 18) [29] & ResNet50 & 87.5 & 93.9 & 91.4 \\
Cross-X (ICCV 19) [19] & ResNet50 & 87.7 & 94.6 & 92.6 \\
DCL (CVPR 19) [6] & ResNet50 & 87.8 & 94.5 & 93.0 \\
TASN (CVPR 19) [34] & ResNet50 & 87.9 & 93.8 & - \\
CIN (AAAI 20) [10] & ResNet50 & 87.5 & 94.1 & 92.8 \\
MC-Loss (TIP 20) [2] & ResNet50 & 87.3 & 93.7 & 92.6 \\
\hline CN-CNN (ours) & ResNet50 & $\mathbf{8 8 . 9}$ & $\mathbf{9 4 . 9}$ & $\mathbf{9 4 . 1}$ \\
\hline \hline
\end{tabular}

Table 2: Comparisons of models with/without the twopathway hierarchy structure. HL: high-low pathway, LH: low-high pathway.

\begin{tabular}{ccc}
\hline Method & Backbone & Accuracy $(\%)$ \\
\hline Baseline & ResNet50 & 84.1 \\
HL & ResNet50 & 86.6 \\
HL+LH & ResNet50 & 87.2 \\
\hline
\end{tabular}

where $\oplus$ represents the operation of addition which uses broadcasting and $M_{i}$ represents the feature maps obtained after HL pathway, i.e., ConvLSTM module.

\subsection{ConvLSTM for Navigation from High-level to Low-level}

Through previous work, we found that the results of some models can be effectively improved by introducing low-level features, even for simple aggregations [27]. Beyond that, we inspired by [14] that ConvlSTM is a very powerful module for connecting and integrating multiple layers of information. Therefore, we consider to introduce CONVLSTM module between multi-layer feature map to help feature fusion between different levels.

In our method, the feature maps of different stages obtained from backbone are first fed into corresponding upsampling operations and $1 \times 1$ convolution layers for obtaining exactly same sizes (i.e., height, width, and channel numbers) of the feature maps. Then they are inputted into ConvLSTM from high-level to low-level. It takes on the role of connecting, guiding and merging different levels of features.

\subsection{Attention Mechanisms for Navigation from Low-level to High-level}

Here, we introduce both spatial and channel attention mechanisms in the navigation from low-level to high-level.

Spatial Attention (SA): As shown in Figure 1, different feature maps of the distinct levels are refined by attentions. The feature
Table 3: Comparisons of models with/without the ConvLSTM Module.

\begin{tabular}{ccc}
\hline Method & Backbone & Accuracy(\%) \\
\hline w/o ConvLSTM & ResNet50 & 87.2 \\
w/ ConvLSTM & ResNet50 & 88.9 \\
\hline
\end{tabular}

maps are first resized into their original size. Then, they are fed into the SA module, respectively, to obtain the SA masks of different levels. The SA module contains one deconvolution operation with $3 \times 3$ kernel. The values of the SA masks are normalized into the interval of $[0,1]$ by Sigmoid function, respectively and they can represent the importance of each region in the specific level.

$$
S A=\sigma\left(\boldsymbol{k} * M_{i}\right)
$$

where $\sigma$ means the Sigmoid function, $*$ refers to the operation of deconvolution and $\boldsymbol{k}$ represents the convolution kernel.

Channel Attention (CA): Similarly, with the feature maps as inputs, the CA masks are obtained by a GAP and two FC layers. The channels of the feature maps of different levels are connected to each other and the information of the lower-level is passed from the bottom to the higher-level.

$$
C A=\sigma(F C 1 \cdot \operatorname{ReLU}(F C 2 \cdot G A P(M i))),
$$

where $F C 1$ and $F C 2$ represents the weights of two fully connected layers, $\sigma$ and $R e L U$ refers to the Sigmoid and the ReLU function, respectively. And GAP refers to the global average pooling operation.

$$
G A P(M i)=\frac{1}{W \times H} \sum_{i=1}^{H} \sum_{j=1}^{W} M_{i}(i, j) .
$$

After obtaining both SA and CA masks, we aggregate them together for a pixel-wise masks and multiply the new masks onto the feature maps obtained by the HL pathway. 


\section{EXPERIMENTAL RESULTS AND DISCUSSION}

We conducted experiments on three common FGVC datasets: CaltechUCSD Birds (CUB-200-2011), Stanford-Cars, and FGVC-Aircraft datasets, which are widely used benchmarks for FGVC.

\subsection{Implementation Details}

We conducted all the experiments on GTX 1080Ti GPU, using Pytorch framework [23]. During training phase, images were resized to $448 \times 448$ which is a general setting. We used ImageNet-pretrained ResNet50 [11] as the backbone model. We took stochastic gradient descent (SGD) as the optimizer and used Batch Normalization for regularization. We trained the network for 300 epochs with initial learning rate as 0.001 for the backbone and 0.1 for the other modules. We set the momentum as 0.9 and the weight decay as $5 \times 10^{-4}$.

\subsection{Comparisons with State-of-the-Art Methods}

Table 1 shows the experiment results on CUB-200-2011, Stanford Cars, and FGVC-Aircraft datasets, trained with ResNet50 as the backbone model. The proposed method I obtains significant improvements on all the three datasets among the referred methods.

The referred methods listed in Table 1 are all weakly supervised methods conducted on the above three datasets. And when using ResNet50 as the backbone model to compare with these methods, the proposed method achieves better performance. In other words, it demonstrates the effectiveness of training networks with the crosslayer navigation. Among them, the proposed method produced especially better results on the CUB-200-2011 dataset that is even more difficult to identify.

\subsection{Ablation Studies}

The ablation studies were conducted on the CUB-200-2011 dataset with the ResNet50 as the backbone model. It aims to find out the impact of each component through comparative experiments.

Impact of the two-pathway hierarchy structure. To compare the performance of the networks with or without the twopathway hierarchy structure, we conducted experiments in the baseline, the HL pathway, and the LH pathway. Here, we simply implemented the HL pathway with directly adding the feature maps together, which is same to the operation in feature pyramidal network [17]. In Table 2, we can find that there is $2.5 \%$ increase in the HL compared with the baseline, which is a significant improvement. Meanwhile, further adding the $\mathrm{LH}$, the accuracy gains another about $0.6 \%$ improvement through the enhanced connection between features of different levels. The accuracies in the table clearly demonstrate the benefits of the two-pathway hierarchy structure.

Impact of the ConvLSTM Module. The difference between the proposed method and baseline ("HL+LH" in Table 2) is that ConvLSTM is introduced. As shown in Table 3, it is obvious that the accuracy obtained with the ConvLSTM is much higher than the baseline, with $1.7 \%$ performance improvement. This indicates that

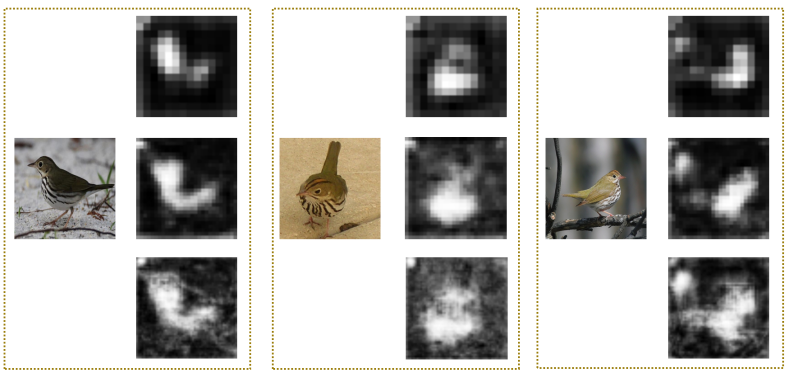

Figure 3: Visualization of the output feature maps of the LH pathway. We randomly select three test images from one specific class on the CUB-200-2011 dataset. The bright white parts in the visualizations is the regions that the network especially pays attention to. In each rectangle, the left color image is the original image. In the right column, from top to bottom, they are high-level to low-level feature maps.

the ConvLSTM can well enhance the correlation between feature maps.

\subsection{Visualizations}

Parts of the visualizations of the attention module are shown in Figure 3. We randomly select three test images from one specific class on the CUB-200-2011 dataset. The bright white parts in the visualizations is the regions that the network especially pays attention to. It can be observed that the low-level information can capture more subtle parts to distinguish the birds. In the higher levels, the network focuses on abstract semantic information more. By integrating low-level information (e.g., colors, edge junctions, and texture patterns), performance can be improved with the enhanced feature representation and the model can accurately locate discriminative regions.

\section{CONCLUSION}

In this paper, we showed that for the FGVC tasks, it is an effective way to improve classification accuracy by using the cross-layer navigation for feature representation enhancement. We propose cross-layer navigation convolutional neural network (CN-CNN), which aims to strengthen the connection between high-level (i.e., semantic information) and low-level (i.e., detailed information) and leverage them for better recognition. Extensive experiments confirmed that the proposed method improves the accuracy on different FGVC datasets. The effectiveness of the components of the proposed method were also discussed. Visualizations can illustrate the ability of the cross-layer navigation.

Acknowledgements: This work was supported in part by the National Key R\&D Program of China under Grant 2019YFF0303300, Subject II No. 2019YFF0303302, and 2020AAA0105200, in part by National Natural Science Foundation of China (NSFC) under Grant 61922015, 61773071, U19B2036, in part by Beijing Natural Science Foundation Project under Grant Z200002.

\section{REFERENCES}

[1] Yuning Chai, Victor Lempitsky, and Andrew Zisserman. 2013. Symbiotic segmentation and part localization for fine-grained categorization. In Proceedings of 
the IEEE International Conference on Computer Vision. 321-328.

[2] Dongliang Chang, Yifeng Ding, Jiyang Xie, Ayan Kumar Bhunia, Xiaoxu Li, Zhanyu Ma, Ming Wu, Jun Guo, and Yi-Zhe Song. 2020. The devil is in the channels: Mutual-channel loss for fine-grained image classification. IEEE Transactions on Image Processing 29 (2020), 4683-4695.

[3] Dongliang Chang, Kaiyue Pang, Yixiao Zheng, Zhanyu Ma, Yi-Zhe Song, and Jun Guo. 2021. Your" Flamingo" is My" Bird": Fine-Grained, or Not. In Proceedings of the IEEE/CVF Conference on Computer Vision and Pattern Recognition. 1147611485 .

[4] Dongliang Chang, Yixiao Zheng, Zhanyu Ma, Ruoyi Du, and Kongming Liang. 2021. Fine-Grained Visual Classification via Simultaneously Learning of Multiregional Multi-grained Features. arXiv preprint arXiv:2102.00367 (2021).

[5] Long Chen, Hanwang Zhang, Jun Xiao, Liqiang Nie, Jian Shao, Wei Liu, and TatSeng Chua. 2017. Sca-cnn: Spatial and channel-wise attention in convolutional networks for image captioning. In Proceedings of the IEEE conference on computer vision and pattern recognition. 5659-5667.

[6] Yue Chen, Yalong Bai, Wei Zhang, and Tao Mei. 2019. Destruction and construction learning for fine-grained image recognition. In Proceedings of the IEEE/CVF Conference on Computer Vision and Pattern Recognition. 5157-5166.

[7] Yifeng Ding, Zhanyu Ma, Shaoguo Wen, Jiyang Xie, Dongliang Chang, Zhongwei Si, Ming Wu, and Haibin Ling. 2021. AP-CNN: weakly supervised attention pyramid convolutional neural network for fine-grained visual classification. IEEE Transactions on Image Processing 30 (2021), 2826-2836.

[8] Ruoyi Du, Dongliang Chang, Ayan Kumar Bhunia, Jiyang Xie, Zhanyu Ma, YiZhe Song, and Jun Guo. 2020. Fine-grained visual classification via progressive multi-granularity training of jigsaw patches. In European Conference on Computer Vision. Springer, 153-168.

[9] Jianlong Fu, Heliang Zheng, and Tao Mei. 2017. Look closer to see better: Recurrent attention convolutional neural network for fine-grained image recognition. In Proceedings of the IEEE conference on computer vision and pattern recognition. $4438-4446$.

[10] Yu Gao, Xintong Han, Xun Wang, Weilin Huang, and Matthew Scott. 2020. Channel Interaction Networks for Fine-Grained Image Categorization. In Proceedings of the AAAI Conference on Artificial Intelligence, Vol. 34. 10818-10825.

[11] Kaiming He, Xiangyu Zhang, Shaoqing Ren, and Jian Sun. 2016. Deep residual learning for image recognition. In Proceedings of the IEEE conference on computer vision and pattern recognition. 770-778.

[12] Shaoli Huang, Zhe Xu, Dacheng Tao, and Ya Zhang. 2016. Part-stacked cnn for fine-grained visual categorization. In Proceedings of the IEEE conference on computer vision and pattern recognition. 1173-1182.

[13] Zixuan Huang and Yin Li. 2020. Interpretable and accurate fine-grained recognition via region grouping. In Proceedings of the IEEE/CVF Conference on Computer Vision and Pattern Recognition. 8662-8672.

[14] Zhongzhan Huang, Senwei Liang, Mingfu Liang, and Haizhao Yang. 2020. Dianet Dense-and-implicit attention network. In Proceedings of the AAAI Conference on Artificial Intelligence, Vol. 34. 4206-4214.

[15] Max Jaderberg, Karen Simonyan, Andrew Zisserman, and Koray Kavukcuoglu. 2015. Spatial transformer networks. arXiv preprint arXiv:1506.02025 (2015)

[16] Jonathan Krause, Michael Stark, Jia Deng, and Li Fei-Fei. 2013. 3d object representations for fine-grained categorization. In Proceedings of the IEEE international conference on computer vision workshops. 554-561.

[17] Tsung-Yi Lin, Piotr Dollár, Ross Girshick, Kaiming He, Bharath Hariharan, and Serge Belongie. 2017. Feature pyramid networks for object detection. In Proceed ings of the IEEE conference on computer vision and pattern recognition. 2117-2125.

[18] Tsung-Yu Lin, Aruni RoyChowdhury, and Subhransu Maji. 2015. Bilinear cnn models for fine-grained visual recognition. In Proceedings of the IEEE international conference on computer vision. 1449-1457.

[19] Wei Luo, Xitong Yang, Xianjie Mo, Yuheng Lu, Larry S Davis, Jun Li, Jian Yang, and Ser-Nam Lim. 2019. Cross-X learning for fine-grained visual categorization. In Proceedings of the IEEE/CVF International Conference on Computer Vision. 82428251.

[20] Zhanyu Ma, Dongliang Chang Jiyang Xie, Yifeng Ding Shaoguo Wen, Xiaoxu Li, Zhongwei Si, and Jun Guo. 2019. Fine-grained vehicle classification with channel max pooling modified CNNs. IEEE Transactions on Vehicular Technology 68, 4 (2019), 3224-3233.

[21] Subhransu Maji, Esa Rahtu, Juho Kannala, Matthew Blaschko, and Andrea Vedaldi. 2013. Fine-grained visual classification of aircraft. arXiv preprint arXiv:1306.5151 (2013).

[22] Jongchan Park, Sanghyun Woo, Joon-Young Lee, and In So Kweon. 2018. Bam: Bottleneck attention module. arXiv preprint arXiv:1807.06514 (2018).

[23] Adam Paszke, Sam Gross, Francisco Massa, Adam Lerer, James Bradbury, Gregory Chanan, Trevor Killeen, Zeming Lin, Natalia Gimelshein, Luca Antiga, et al. 2019. Pytorch: An imperative style, high-performance deep learning library. arXiv preprint arXiv:1912.01703 (2019).

[24] Ming Sun, Yuchen Yuan, Feng Zhou, and Errui Ding. 2018. Multi-attention multiclass constraint for fine-grained image recognition. In Proceedings of the European Conference on Computer Vision (ECCV). 805-821.
[25] Catherine Wah, Steve Branson, Peter Welinder, Pietro Perona, and Serge Belongie. 2011. The caltech-ucsd birds-200-2011 dataset. (2011)

[26] Yaming Wang, Vlad I Morariu, and Larry S Davis. 2018. Learning a discriminative filter bank within a cnn for fine-grained recognition. In Proceedings of the IEEE conference on computer vision and pattern recognition. 4148-4157.

[27] Lior Wolf and Stanley Bileschi. 2006. A critical view of context. International Journal of Computer Vision 69, 2 (2006), 251-261.

[28] Tianjun Xiao, Yichong Xu, Kuiyuan Yang, Jiaxing Zhang, Yuxin Peng, and Zheng Zhang. 2015. The application of two-level attention models in deep convolutional neural network for fine-grained image classification. In Proceedings of the IEEE conference on computer vision and pattern recognition. 842-850.

[29] Ze Yang, Tiange Luo, Dong Wang, Zhiqiang Hu, Jun Gao, and Liwei Wang. 2018. Learning to navigate for fine-grained classification. In Proceedings of the European Conference on Computer Vision (ECCV). 420-435.

[30] Ning Zhang, Jeff Donahue, Ross Girshick, and Trevor Darrell. 2014. Part-based R-CNNs for fine-grained category detection. In European conference on computer vision. Springer, 834-849.

[31] Tian Zhang, Dongliang Chang, Zhanyu Ma, and Jun Guo. 2021. Progressive Co-Attention Network for Fine-grained Visual Classification. arXiv preprint arXiv:2101.08527 (2021).

[32] Yu Zhang, Xiu-Shen Wei, Jianxin Wu, Jianfei Cai, Jiangbo Lu, Viet-Anh Nguyen, and Minh N Do. 2016. Weakly supervised fine-grained categorization with partbased image representation. IEEE Transactions on Image Processing 25, 4 (2016), 1713-1725.

[33] Heliang Zheng, Jianlong Fu, Tao Mei, and Jiebo Luo. 2017. Learning multiattention convolutional neural network for fine-grained image recognition. In Proceedings of the IEEE international conference on computer vision. 5209-5217.

[34] Heliang Zheng, Jianlong Fu, Zheng-Jun Zha, and Jiebo Luo. 2019. Looking for the devil in the details: Learning trilinear attention sampling network for fine-grained image recognition. In Proceedings of the IEEE/CVF Conference on Computer Vision and Pattern Recognition. 5012-5021. 